\title{
POTENCIANDO IDEAS: UNA PROPUESTA DE ANÁLISIS PARA DISCUTIR EL DESARROLLO DE TECNOLOGÍA NUCLEAR EN ARGENTINA Y MÉXICO (1950-1991)
}

\author{
ADVANCING IDEAS: A PROPOSAL FOR ANALYSIS OF \\ THE DEVELOPMENT OF NUCLEAR TECHNOLOGY IN \\ ARGENTINA AND MEXICO (1950-1991)
}

\author{
RENFORCER LE DÉBAT D'IDÉES: PROPOSITION \\ D'ANALYSE EN FORME DE CONTRIBUTION AU DÉBAT \\ SUR LE DÉVELOPPEMENT DE LA TECHNOLOGIE \\ NUCLÉAIRE EN ARGENTINE ET AU MEXIQUE (1950-1991)
}

\author{
Nevia Vera \\ CEIPIL-UNICEN-CONICET \\ mneviavera@gmail.com
}

Resumen: Este artículo presenta un análisis comparativo basado en el método de rastreo de proceso de los programas nucleares de Argentina y México entre 1950 y 1991. Estudia los niveles de avance de cada caso con base en la posibilidad de implementación de ideas de autonomía tecnológica, de acuerdo con su capacidad de potenciación determinada por tres dimensiones: la autonomía lateral de la principal agencia nuclear, la formación de alianzas estratégicas con actores intermediarios clave y una política exterior en materia nuclear conducente a tal meta.

Palabras clave: Argentina; México; tecnología nuclear; autonomía tecnológica; ideas.

ABSTRACT: This article presents a process tracing-based comparative analysis of the nuclear programs in Argentina and Mexico between 1950 and 1991. It seeks to study the development of each case according to the possibility of implementing the idea of technological autonomy, based on their capacity for advancement as determined by three specific dimensions: the lateral autonomy of the main nuclear agency, the formation of strategic alliances with key intermediary actors, and a foreign policy in nuclear matters conducive to this goal. 
Keywords: Argentina; Mexico; nuclear technology; technological autonomy; ideas.

\section{Traducción de Fionn Petch, CM Idiomas}

RÉSumé: cet article offre une analyse comparative ayant pour méthode un passage en revue du processus suivi par les programmes nucléaires argentin et mexicain entre 1950 et 1991. On y examine le degré d'avancement de chacun des deux cas, déterminé par la possibilité d'une mise en pratique de principes d'autonomie technologique dont on évaluera le potentiel de développement à l'aune de trois facteurs: l'autonomie latérale dont a disposé la principale agence nucléaire de chacun des deux pays, la mise en place d'alliance stratégiques avec des acteurs intermédiaires clés, et une politique étrangère nucléaire orientée vers l'objectif fixé.

Mots clés: Argentine; Mexique; technologie nucléaire; autonomie technologique; idées.

Traducción de Ariel Elbaz, CM IDiomas

Fecha de recepción: junio de 2019

Fecha de aceptación: octubre de 2020 


\section{INTRODUCCIÓN}

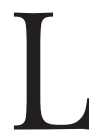

a ciencia y la tecnología (CyT) se han convertido en los motores de cambio, desarrollo y dinamización de las estructuras económicas, políticas y sociales tanto dentro de los Estados como entre ellos. ${ }^{1}$ Los avances que en este ámbito han tenido lugar desde el siglo xxi han impactado en la capacidad física y de interacción del sistema internacional de tal forma que el escenario mundial del siglo xx se caracterizó por presentar una enorme brecha en los niveles de poder estatal absoluto y relativo, generando su mayor concentración en unos pocos Estados centrales. ${ }^{2}$

En el siglo $\mathrm{xx}$, el fenómeno anteriormente descrito se intensificó con la introducción de la tecnología nuclear, entre otras. Ésta ha sido clave no sólo debido al amplio abanico de usos civiles que ofrece -en las áreas industrial, medicinal y energética-, sino fundamentalmente porque puede orientarse hacia la fabricación de armas de destrucción masiva, lo que se ha transformado desde la Segunda Guerra Mundial en factor de disuasión, jerarquía y prestigio, así como en garantía de integridad física e independencia para los Estados en el sistema internacional. ${ }^{3}$

Durante la Guerra Fría, la cooperación internacional en materia de tecnología nuclear civil creció amparada en el programa "átomos para la paz", patrocinado por Estados Unidos, cuyo objetivo fue brindar asistencia técnica y financiera a países en desarrollo (PED) para poder acceder a la era nuclear. Al mismo tiempo, éstos pretendieron encontrar un nicho comercial que permitiera amortizar las ingentes inver-

${ }^{1}$ John Krige, John y Kai-Henrik Barth, "Introduction: Science, Technology, and International Affairs", Osiris, 21, núm. 1 (2006), pp. 1-21.

2 Ver Barry Buzan y George Lawson, The Global Transformation: History, Modernity, and the Making of International Relations, Cambridge, University Press.

${ }^{3}$ Véase Robert Gilpin, War and change in world politics, Cambridge, University Press, 1981. 
siones efectuadas en tecnología atómica. Muchos PED, como Argentina y México, se embarcaron en tales programas entusiasmados por la expectativa de desarrollo que traía consigo la tecnología nuclear. ${ }^{4}$ Sin embargo, sus programas atómicos alcanzaron distintos niveles de éxito.

¿Qué explica que dos países con similar potencial para desarrollar su sector nuclear obtengan avances dispares luego de transcurrido determinado tiempo? Partiendo de esta pregunta, el presente artículo busca explicar los resultados disímiles en los avances de los programas atómicos de Argentina y México entre los años 1950 y 1991, enfatizando la importancia que ciertas ideas, como la de autonomía tecnológica, pueden tener a la hora de conferir un cariz diferente a las políticas de desarrollo de tecnología nuclear, e identificando factores que hicieron posible su traducción en políticas públicas.

Se sostiene que, en los casos estudiados, la diferencia en el desarrollo alcanzado radicó en la capacidad que tuvo la comunidad de expertos nucleares en Argentina para potenciar la idea de autonomía tecnológica, aprovechando la autonomía lateral de la Comisión Nacional de Energía Atómica, CNEA, forjando una alianza con actores claves como las Fuerzas Armadas y con una política exterior de semialineación con el régimen de no proliferación, todo lo cual resultó en mayores avances. Por su parte, en el caso de México, el rezago del sector no se explica por la ausencia de estas ideas, sino por su incapacidad de concretarse en políticas públicas efectivas debido a la poca autonomía lateral de la Comisión Nacional de Energía Nuclear, CNEN, la falta de alianzas duraderas y estables con actores claves y una política exterior de alineación con el régimen de no proliferación.

La elección de casos responde a que ambos son países latinoamericanos que comparten varias características: cuentan con ciertos niveles de industrialización, aunque dependen en gran medida de la exportación de productos

${ }^{4}$ Véase Rodney Jones, "Atomic Diplomacy in Developing Countries", Journal of International Affairs, 34, núm. 1 (1980), pp. 89-117. 
primarios; son países de ingresos medios y de un tamaño considerable, con cierto peso en la región; han tenido la capacidad de comprometerse efectivamente en acciones e iniciativas diplomáticas, de participar activamente en las instituciones internacionales, y de movilizar coaliciones regionales en ciertos foros multilaterales. ${ }^{5}$

Sin embargo, a pesar de haber comenzado sus programas nucleares en la década de 1950 con el objetivo compartido de lograr autonomía tecnológica, se ubican en dos extremos opuestos en el continuum imaginario de los avances nucleares de Latinoamérica. Podría decirse que son dos casos diversos con cuya elección se busca ilustrar la mayor variación de las dimensiones planteadas, donde las diferentes combinaciones posibles de los valores de cada variable tienen un efecto concreto en los resultados analizados. ${ }^{6}$ De esta forma, el método comparativo cualitativo y el de rastreo de proceso ${ }^{7}$ se transforman en herramientas útiles para analizar los casos de estos dos países, cuyos sectores nucleares fueron concebidos para objetivos similares y que, sin embargo, discurrieron por caminos distintos que resultaron en trayectorias prácticamente opuestas.

El periodo histórico seleccionado (1950-1991) responde a la decisión de analizar estos desarrollos durante la Guerra Fría, lapso dentro del cual se originaron las respectivas instituciones nucleares. En Argentina, la política nuclear oficial se estableció con la creación de la cNEA, organización que alcanzó sus mayores avances en las poco más de tres décadas

${ }^{5}$ Véase Arturo Sotomayor, U.S.-Latin American Nuclear Relations: From Commitment to Defiance, California, US Naval postgraduate School, 2012.

${ }^{6}$ Véase John Gerring, Case study research. Principles and practices, Cambridge, University Press.

${ }^{7}$ Este método consiste en la aplicación de una examinación cuidadosa y profunda de los casos, para encontrar mecanismos causales que permitan poner de relieve secuencias para una mejor comprensión de la actuación de las variables propuestas. Para un análisis más detallado de este método véase David Collier, "Understanding Process Tracing”, PS: Political Science and Politics 44, núm. 4 (octubre de 2011), pp. 823-830. 
subsiguientes. Por su parte, México comenzó sus desarrollos en el terreno de la tecnología atómica a partir de la creación de la CNEN, en 1956, pero no ha alcanzado hitos tecnológicos comparables a los argentinos (ver cuadro 1 ).

\section{CUADro 1}

Niveles de desarrollo nuclear de Argentina y México hacia 1991

\begin{tabular}{ccc}
\hline & Argentina & México \\
Reactores nacionales & 6 (5 de fabricación & 3 (importados \\
nacional) & o donados) \\
Reactores de potencia en marcha & 2 & 1 \\
Capacidad de enriquecimiento del Uranio & Sí & No \\
Fabricación de radioisótopos & Sí & Sí \\
Reactores exportados & 5 & 0 \\
Empresas del sector nuclear & 6 & 0 \\
\hline
\end{tabular}

Fuente: elaboración propia en base a datos de CNEA y del Instituto Nacional de Investigaciones Nucleares, ININ.

La literatura comparativa sobre el desarrollo nuclear de Argentina y Brasil es abundante, pues ambos países han despertado curiosidad al representar ejemplos del desarrollo de tecnología estratégica de punta en un contexto de PED, inestables económicamente y con democracias frágiles, factores limitantes del progreso de políticas públicas duraderas y coherentes a lo largo del tiempo. Sin embargo, ambos lograron erigir al desarrollo nuclear en política de Estado. No obstante, en el caso de México, encontrar información sobre su desarrollo nuclear es más difícil, especialmente si se buscan análisis comparativos. ${ }^{8}$

${ }^{8}$ Una excepción lo constituyen los trabajos de Arturo Sotomayor, op. cit., y Arturo Sotomayor, "Brazil and Mexico in the Nonproliferation Regime, common structures and divergent trajectories in Latin America", en Jeffrey Fields (ed.), State Behavior and the Nuclear Nonproliferation Regime, EEuU, University of Georgia Press, 2014, 340 pp. 
Como lo demuestran varios estudios sobre los casos específicos de Argentina y Brasil, ${ }^{9}$ los factores materiales no bastan para explicar los avances o retrocesos en el desarrollo de este tipo de tecnología, y como intentará demostrarse, en el caso de México tampoco, o al menos no son suficientes. Además, al ser dual, la tecnología atómica incorpora una arista de complejidad que rebasa el análisis meramente económico: su desarrollo siempre ha sido fuertemente regulado a través del régimen internacional de no proliferación. Para los PED, como los dos analizados en este artículo, lo anterior influyó en dos sentidos: por un lado, implicó amplias restricciones para acceder a insumos atómicos, lo cual condicionó en cierta forma sus opciones tecnológicas; por otro, generó respuestas diversas frente a los mencionados regímenes, que a su vez incidieron en desarrollos locales posteriores.

A partir de lo anterior, es necesario explicitar los supuestos teóricos que orientan la investigación. En primer lugar, el artículo sostiene que los mayores avances se registraron allí donde fue posible implementar políticas conducentes al logro de la autonomía tecnológica. La influencia de esta idea existió en ambos casos al delinear las políticas nucleares de cada país (aunque en México fue denominada autodeterminación tecnológica). Pero, como se reconoce que las ideas suelen ser subsidiarias de otras explicaciones ${ }^{10}$ (es decir, no pueden explicar por sí solas la variación de resultados), se pretende analizar, en segundo lugar, si esta idea presente en am-

${ }^{9}$ Véase Emanuel Adler, "State Institutions, Ideology, and Autonomous Technological Development: Computers and Nuclear Energy in Argentina and Brazil”, Latin American Research Review, vol. 23, núm. 2 (1987), pp. 59-90; Etel Solingen, "Macropolitical Consensus and Lateral Autonomy in Industrial Policy: The Nuclear Sector in Brazil and Argentina”, International Organization, 47, núm. 2 (1993), pp. 263-298; Jacques Hymans, "Of gauchos and gringos: Why Argentina never wanted the bomb, and why the United States thought it did”, Security Studies, 10 núm. 3 (2001), pp. 153-185, y Rodrigo Mallea, Matías Apektor y Nicholas Wheeler, Origens da cooperação nuclear. Uma historia oral critica entre Argentina e Brasil, Río de Janeiro, FGV, Escola de Ciências Sociais, 2012.

${ }^{10}$ Solingen, art. cit. 
bos casos pudo traducirse en una política pública, de acuerdo con los alcances y límites que en ella imprimieron tres dimensiones principales: la autonomía lateral de las comunidades científicas nucleares congregadas en torno a las instituciones centrales de cada entramado nuclear, su vinculación con actores intermediarios clave y la política exterior en materia nuclear de cada país. En consecuencia, busca desentrañar si fue posible que la idea de autonomía tecnológica se materializara en las prácticas de los expertos, ingenieros, físicos, científicos y burócratas del sector, según haya sido potenciada o limitada por cada una de esas dimensiones para explicar los niveles de desarrollo nuclear divergentes entre ambos países.

Para ello, en el apartado siguiente se describen las tres dimensiones que, se sostiene, influyeron en la capacidad de concreción de los postulados de la idea de autonomía tecnológica; luego se analiza cada uno de los casos prestando atención a estas dimensiones en particular y, por último, se presentan las reflexiones finales.

DimenSIONES DE LA POTENCIACIÓN DE IDEAS: AUTONOMÍA, ALIANZAS Y POLÍTICA EXTERIOR

La idea de autonomía o autodeterminación tecnológica planteada aquí se originó como parte de un cuerpo de pensamiento denominado pensamiento latinoamericano en ciencia, tecnología y sociedad (PLACTS) que tuvo relevancia entre los años 1950 y 1970. Sus principales pensadores fueron Amílcar Herrera, Jorge Sábato y Oscar Varsavsky, Helio Jaguaribe, Máximo Halty Carrére, Francisco Sagasti, Carlos Martínez Vidal y Miguel Wionczek, entre otros. ${ }^{11}$ Tuvo como principal objetivo pensar la ciencia sin separarla de su marco de concepción,

${ }^{11}$ Carlos Martínez Vidal y Manuel Marí, "La escuela latinoamericana de pensamiento en ciencia, tecnología y desarrollo. Notas de un proyecto de investigación", Revista iberoamericana de ciencia, tecnología, sociedad e innovación, núm. 4 (septiembre-diciembre de 2002), https://www.oei.es/ historico/revistactsi/numero4/escuelalatinoamericana.htm 
la sociedad, y por lo tanto sin desviar la mirada de la situación de dependencia de Latinoamérica respecto a la cyт. Partió de identificar asimetrías entre aquellos países productores de materias primas y las economías centrales, demandantes de productos primarios y exportadores de bienes industriales con alto contenido tecnológico, lo cual generaba una constante dependencia económica y tecnológica de los PED, que al no poder escapar de tal estructura se encontraban casi condenados al subdesarrollo.

Esta idea es una de las más importantes de este cuerpo de pensamiento: implicó abogar por un proceso de maduración tecnológica de los países que debería comenzar con una autonomía decisional tecnológica propia, y comprender la capacidad de manejo de tecnologías, formulación de su demanda, selección de tecnologías y alternativas tecnológicas para no estar atados a monopolios, desagregación de paquetes tecnológicos (apertura de cajas negras), su rearmado y adaptación teniendo en cuenta necesidades locales; capacidad de negociación y compra a los proveedores según se crea conveniente, y finalmente, desarrollo íntegro de forma local con capacidades propias. ${ }^{12}$

Por lo tanto, gran parte de las críticas de esta corriente se centraron en la adopción de tecnología foránea sin una evaluación de las capacidades y contextos locales. En México, su mayor representante, Wionczek, criticó la estrategia de adquisición de tecnología en varios PED por medio de la radicación de filiales de empresas multinacionales y la posterior compra de cajas negras tecnológicas. En Argentina, su máximo exponente, Sábato, promovió un modelo de diseño de políticas científicas y tecnológicas conocido como el Triángulo de Sábato, que proponía el desarrollo de proyectos tecnológicos a través de la vinculación estratégica de tres actores

12 Renato Dagnino, Hernán Thomas y Amílcar Davyt, "El pensamiento en ciencia, tecnología y sociedad en Latinoamérica: una interpretación política de su trayectoria”, Revista REDES, 3, núm. 7 (septiembre de 1996), pp. 13-53; Vidal y Marí, op. cit. 
principales: el sector productivo, el Estado, como generador de políticas tecnológicas y otorgante de financiamiento, y el sector científico-tecnológico.

En este artículo se considera la idea de autonomía tecnológica como una idea causal -es decir, como el tipo de idea que provee una guía a la acción política, sugiere soluciones a un problema y otorga una base de acción para el diseño e implementación de políticas públicas, ${ }^{13}$ asumiendo que las decisiones de ciertos actores se toman considerando estructuras cognitivas moldeadas por compromisos normativos, creencias, modelos mentales, que influyen en sus prescripciones políticas. ${ }^{14}$

Pero como se reconoce que las ideas por sí solas no bastan para otorgar explicaciones cabales de los fenómenos, es necesario analizar cómo se vehiculizan o potencian hasta materializarse en políticas concretas y, por lo tanto, cómo logran seducir a las elites políticas decisoras, lo que en definitiva les otorga visibilidad en la agenda pública. La primera dimensión considerada es la autonomía lateral de la principal agencia encargada del desarrollo de la actividad nuclear (la CNEA para Argentina y la CNEN para México) con respecto a otras instituciones, lo que implica menos competencia interagencias y mayor concentración de tareas y capacidad de ejecución en un solo ente. Cuanto mayor es el monopolio de actividades y responsabilidades de tal organización con respecto a los aspectos de determinado programa (desde planificación, financiamiento, negociaciones, formación de recursos humanos, etc.), mayor es su autonomía lateral y, por lo tanto, su protección frente a las presiones y la competencia de otras agencias del Estado es mayor. ${ }^{15}$ Lo opuesto a la

13 Vivien Schmidt, "Discursive Institutionalism: The Explanatory Power of Ideas and Discourse", Annual Review of Political Science, 11 (2008), pp. 303-326.

${ }^{14}$ Alan Jacobs, "Process-tracing the effect of ideas", en Andrew Bennett y Jeffrey T. Checkel (eds.), Process Tracing in the Social Sciences: From Metaphor to Analytic Tool, Cambridge, University Press, 2014.

${ }^{15}$ Solingen, art. cit. 
autonomía lateral de las agencias es la segmentación lateral, donde varios entes tienen jurisdicciones yuxtapuestas en un mismo ámbito. ${ }^{16}$

La importancia de esta dimensión radica en que permite establecer la capacidad de la comunidad científica nuclear que representa para influir en la aprobación de sus proyectos, y tomar decisiones sobre su implementación, sobre todo si se tiene en cuenta que los atributos de autonomía fueron propios de un conjunto de agencias estatales desde la Segunda Guerra Mundial, lo que permitió que crecieran y se estabilizaran manteniendo gran coherencia interna aun en contextos de baja estatalidad, y que además tuvieran gran capacidad de definición de agenda en el ámbito público. ${ }^{17}$ Empero, no hay que dejar de lado que esta relación implica un problema de asimetría de la información para el Estado ya que, para tomar decisiones, depende de un actor (la agencia nuclear) con sus propios proyectos e ideas, no siempre convergentes con los suyos. Esto pone en evidencia que cuanto mayor sea la autonomía de una agencia (nuclear en este caso), mayor será su posibilidad de limitar las opciones y elecciones tecnológicas presentadas a las esferas decisoras y, por lo tanto, dada esta asimetría de información, mayor será la posibilidad de que estas agencias nucleares logren alcanzar sus objetivos tecnológicos.

A partir de lo anterior se desprende que cuanta mayor es la autonomía lateral de una agencia, mayor es su posibilidad de elevar a las esferas decisoras un abanico limitado de opciones tecnológicas conducentes a la materialización de los propios proyectos. Por lo tanto, se espera que determinadas agencias tengan mayor posibilidad de imponer sus pro-

${ }^{16}$ Ibid. Véase también Oscar Oszlak, "Políticas públicas y regímenes políticos. Reflexiones a partir de algunas experiencias latinoamericanas", Documento de estudio de CEDES, 3, núm. 2, Buenos Aires, 1984.

${ }^{17}$ Julián Gadano, "El desarrollo nuclear pacífico argentino: factores que lo hicieron posible y algunas hipótesis sobre su futuro", Flacso-ISA. Joint International Conference "Global and regional powers in a changing world", Buenos Aires, 10 de julio de 2014, pp. 47-66. 
pios proyectos cuanta mayor sea su autonomía lateral (como sucedió con CNEA). Por el contrario, se espera que ello sea más difícil en contextos de segmentación lateral donde dos o más agencias tengan que competir por imponer sus proyectos tecnológicos (caso de CNEN).

La segunda dimensión es aquella sobre las alianzas o vinculaciones con actores intermediarios clave capaces de vehiculizar los proyectos nucleares diseñados por los expertos del sector. En el caso de Argentina, las Fuerzas Armadas, en particular la Marina, se convirtieron en un actor intermediario clave por varios motivos: en primer lugar, por la existencia de gobiernos militares en el país, lo que les otorgó poder de decisión en materia de políticas públicas, entre ellas, la nuclear. En segundo lugar, por su interés específico en el desarrollo de tecnología atómica, no tanto por sus fines bélicos sino por ser un elemento eminentemente simbólico de primacía regional, sobre todo frente a Brasil, rival importante al menos hasta la década de 1980. Por su parte, en México las Fuerzas Armadas fueron relegadas por completo de cualquier decisión nuclear, razón por la cual no se convirtieron en actores de peso en cuestiones de tecnología atómica. Más bien, las alianzas de los expertos nucleares mexicanos se orientaron hacia la formación de redes científicas internacionales y regionales y vínculos domésticos ad hoc.

La tercera y última dimensión es la de la política exterior en materia nuclear sostenida durante el periodo en cuestión, y es importante porque el periodo elegido comprende el lapso en que la disputa de poder entre la Unión Soviética y Estados Unidos en el marco de la Guerra Fría resultó en la negociación de un régimen internacional de no proliferación que implicó fuertes restricciones al desarrollo de tecnología nuclear autónoma para los PED. Dunn ${ }^{18}$ arroja luz sobre la importancia de tomar en cuenta la incidencia del régimen de no proliferación y la relevancia de las respuestas de política exterior, al

18 Lewis Dunn, "The NPT", The Nonproliferation Review, 16, núm. 2 (2009), pp. 143-172. 
señalar que aquellos países que se adhirieron al régimen antes de comenzar sus proyectos nucleares tuvieron más dificultades para llevarlos a buen término en un lapso prudente. Aunque el autor analiza programas bélicos, su hipótesis permite entender la desconfianza de varios países con respecto al efecto del régimen en los programas atómicos nacionales.

En tal marco, la política exterior sirvió para responder a este contexto internacional crecientemente restrictivo. $\mathrm{Si}$ bien ambos países estuvieron en la esfera de influencia de Estados Unidos, las relaciones con éste no fueron las mismas para ambos, como tampoco lo fueron los efectos de la mayor cercanía de México vis à vis la distancia de Argentina. Todo lo anterior derivó en dos posturas divergentes: aquella de completa adhesión al régimen, por parte de México, y aquella de semialineamiento, de Argentina. A partir de las siguientes secciones se analiza cómo cada una de las dimensiones anteriores permitió o limitó la vehiculización de las ideas de autonomía tecnológica para su materialización en resultados concretos en los respectivos sectores nucleares.

\section{El caso de Argentina}

\section{Autonomía lateral}

La principal agencia del entramado nuclear argentino, la CNEA, creada en mayo de 1950, centralizó desde un principio todas las tareas referentes al ámbito atómico, desde la prospección de uranio, hasta la planificación de las políticas tecnológicas en materia nuclear en general, pasando por la formación de sus propios recursos humanos. Esto permitió que la organización se mantuviera al margen de los avatares políticos ${ }^{19} \mathrm{y}$ que no se viera forzada a competir con agencias similares, por

${ }^{19}$ Adler, art. cit.; Solingen, art.cit. Véase también Julio Carasales, $L a$ cooperación internacional de la Argentina en el campo nuclear, Buenos Aires, CARI, 1999. 
lo que pudo permanecer al margen de la competencia partidaria y de lobbies contrarios como el de la energía hidroeléctrica con sus respectivos aliados en la Secretaría de Energía. ${ }^{20}$

Esta autonomía lateral le dio libertad a CNEA para seguir el camino que consideró más conducente hacia la autonomía tecnológica, que en la práctica se materializó en la aplicación de políticas cuidadosamente diseñadas de transferencia de tecnología, adaptación, generación de proveedores locales, formación de recursos humanos, actividades de I+D y creación de una infraestructura de laboratorios y centros de investigación. ${ }^{21}$

Esta orientación sustentada en la aspiración de autonomía tecnológica también se hizo presente a la hora de elegir el tipo de tecnología que tendrían las futuras centrales de potencia. Aun cuando Argentina no contaba todavía con las capacidades necesarias para construir centrales nucleares de forma endógena en su totalidad, la decisión siempre estuvo orientada al desarrollo de capacidades locales. ${ }^{22}$ Así, la decisión de 1969 de comprar un reactor de potencia para la central nuclear Atucha I a base de uranio natural y agua pesada a Siemens, empresa de la República Federal de Alemania (RFA), se basó en dos cuestiones clave: por un lado, permitía una mayor participación de industrias nacionales y mayor transferencia de tecnología; por otro, tenía ventajas geopolíticas. Con respecto a lo primero, se organizó un comité llamado Grupo de Industrias Nacionales (GIN), que determinó que la industria local podría contribuir con $33 \%$ de los insumos para la planta. Esta cifra buscó elevarse en la construcción de la segunda planta (Embalse), en la cual la participación de las empresas locales llegó a $58 \% .^{23}$

Tales elecciones obraron en favor de una mayor independencia tecnológica en las consideraciones geopolíticas: optar

${ }^{20}$ Adler, art. cit.

${ }^{21}$ Incluso se la llegó a conocer como Comisión Nacional de Educación Atómica. Véase Adler, art. cit.

22 Véase Hymans, art. cit.

23 Adler, art. cit. 
por tecnología de uranio natural y agua pesada en lugar de uranio enriquecido respondió también a que este último, considerado material de uso dual, se regía por una lógica oligopólica, para evitar el riesgo de que fuera utilizado para fines bélicos. En ese entonces, los principales exportadores del combustible eran Estados Unidos y la Unión Soviética, y ambos basaban sus ventas en la firma de tratados como el de No Proliferación (TNP) o el de Tlatelolco ( $\mathrm{TdT}$ ), que, en la visión de los funcionarios y científicos argentinos, obstaculizaban los desarrollos de tecnología nuclear propia, que era justamente la meta principal de la CNEA.

De esta forma, la opción de reactores de agua pesada y uranio natural (mineral con el que además Argentina contaba en su propio territorio) abría paso al camino de la autonomía, aunque lo retrasaba un poco, y si bien implicaba una corta dependencia de agua pesada importada (cuya venta provenía de varios países, no de un monopolio u oligopolio), se esperaba que tal situación se mantuviera sólo hasta poder desarrollar una planta de procesamiento de agua pesada propia.

\section{Alianzas con actores intermediarios clave}

Como se mencionó en párrafos anteriores, en el caso argentino uno de los aliados más importantes del sector nuclear estaba integrado por las Fuerzas Armadas, más específicamente, la Marina. La cNEA estuvo bajo su protección desde 1952, luego del fiasco del Affaire Richter, ${ }^{24}$ lo que también influyó en el fuerte liderazgo ejercido por algunos cuadros técnicos en su interior y su continuidad a lo largo de años, ${ }^{25}$

${ }^{24}$ Para un relato pormenorizado sobre este suceso, véase Diego Hurtado, El sueño de la Argentina atómica. Política, tecnología nuclear y desarrollo nacional (1945-2006), Buenos Aires, Editorial Edhasa, 2014.

${ }^{25}$ Así, mientras la sociedad vivía las convulsiones políticas típicas de la inestabilidad democrática, la CNEA sólo tuvo cuatro presidentes en treinta años (el almirante Oscar Quihillalt, por ejemplo, vio dejar el 
ya que esto permitió que la institución tomara sus propias decisiones estratégicas. ${ }^{26}$

Parte de la explicación de esta alianza radica en que el presidente Juan D. Perón (quien gobernó Argentina entre 1946 y 1955, y más tarde entre 1973 y 1974), elegido democráticamente pero de extracción militar, apoyó fuertemente el desarrollo de industrias estratégicas con una visión autonomista, correspondiente a sus concepciones militares, visión coincidente con la de muchos científicos de la CNEA.

Por otra parte, aunque los gobiernos militares tuvieron un rol importante en la continuidad del apoyo al programa nuclear, éste no fue siempre el caso. Sin ir más lejos, durante la última dictadura militar en Argentina (1976-1983) se tomaron las primeras medidas que derivaron en el principio de la desindustrialización del país. Sin embargo, y gracias a negociaciones con sectores desarrollistas de las Fuerzas Armadas, resabios disminuidos de épocas anteriores, el sector nuclear se mantuvo a salvo. Sin duda, la impronta que el peronismo le dio a la industria en sus comienzos fue fundamental y, seguramente, fue importante que la cNEA haya estado bajo la protección del sector militar más nacionalista o desarrollista (heredero de esa concepción peronista de la necesidad de autonomía y autoabastecimiento energético), aun en contextos en los que se promovían políticas desindustrializantes y en los que la CYT no fueron prioridad.

Por lo tanto, puede decirse que la supervivencia del área nuclear, aun en contexto de implementación de políticas desindustrializantes, estuvo fuertemente relacionado con el hecho de haber ocupado un lugar tan preponderante en el imaginario militar desde la década de 1940, como sector vinculado en forma directa a las soluciones para los problemas del desarrollo y la industrialización, clave para los sectores militares desarrollistas.

mandato a ocho presidentes durante los dieciocho años al frente de la institución).

${ }^{26}$ Adler, art. cit.; Solingen, art. cit.; Hurtado, op.cit. 


\section{Política exterior argentina en materia nuclear}

La política exterior argentina en materia nuclear fue consistente a lo largo del periodo bajo análisis. Ésta se desplegó en dos ámbitos principales: por un lado, el regional, donde el país buscó ser líder en materia nuclear para poder posicionarse como proveedor de materiales nucleares y, por otro, en un nivel multilateral, correspondiente a los principales foros internacionales que formaban parte del régimen de no proliferación, manteniendo una postura contraria a los privilegios tecnológicos de los países nuclearmente armados y a las restricciones de acceso y desarrollo de tecnología.

Con respecto a esto último, es necesario aclarar que la postura argentina no fue de completo rechazo: el país se adhirió a la Organización Internacional de Energía Atómica (OIEA) desde su fundación en 1957; había demostrado su vocación pacífica cuando propuso el artículo $\mathrm{V}$ del Tratado Antártico (que prohibía explosiones nucleares en aquel territorio), y había sido uno de los primeros países en firmar el Tratado de prohibición parcial de ensayos nucleares en la atmósfera, firmado en Moscú. Pero rechazó desde un principio el $\mathrm{TdT}^{27}$ (1967), y el TNP 28 (1968), que para los funcionarios argentinos implicaba "el desarme de los desarmados". ${ }^{29}$

${ }^{27}$ El Tratado de Tlatelolco buscó prohibir la fabricación de armas nucleares en la región, y el establecimiento de un sistema de control basado en la aplicación de las salvaguardias del orea, la no introducción de armas nucleares en la región y la prohibición del tránsito de las mismas.

${ }^{28}$ Los objetivos del TNP fueron: el compromiso de los Estados poseedores de armas nucleares de no transferir, alentar y/o contribuir a la fabricación de armas nucleares o dispositivos explosivos; el compromiso, de los Estados no poseedores de armas nucleares de no aceptar transferencias ni contribuciones por parte de los Estados poseedores de armas nucleares para la fabricación de armas atómicas o dispositivos explosivos, y el acuerdo de impedir la desviación de material nuclear hacia usos bélicos, en cooperación con el OIEA, supeditándose además a su sistema de salvaguardias. Finalmente, debía trabajarse conjuntamente en pos del desarme y el fin de la carrera armamentista.

${ }^{29}$ Carasales, op. cit. 
Los argumentos que defendió Argentina para rechazar la ratificación de los tratados se basaron en que ambos planteaban un statu quo que perpetuaba una situación desigual, con un reducido grupo de países poseedores de armas nucleares y la restante mayoría que renunciaba a ellas de forma voluntaria, además de dificultar el desarrollo de tecnología propia para industrializar y modernizar sus respectivos territorios. ${ }^{30}$ Si bien el artículo IV del TNP garantizaba el derecho de los países a sus propios desarrollos nucleares, la batería de condiciones impuestas dificultaba tales procesos. El representante argentino ante la Organización de Naciones Unidas (ONU), José María Ruda, defendió la posición del país en la Asamblea General de la organización, en 1968, alegando que para Argentina era importante evitar que el Tratado pudiera suponer un obstáculo al propio desarrollo, por una parte y, por otra, que constituyera la base jurídica para la imposición explícita de la dependencia tecnológica. ${ }^{31}$

El régimen de no proliferación se endureció luego de que la India hiciera detonar un artefacto nuclear propio en 1974, lo que originó la creación del Grupo de Proveedores Nucleares (GPN), formado por aquellos países autorizados a exportar material y tecnología nuclear, y promotor de condicionamientos extras para permitir el intercambio de material atómico. El organismo dificultó la provisión de insumos importados por Argentina: AECL, la empresa canadiense que debía proveer la segunda central atómica, ejerció mayores presiones con respecto a las salvaguardas, y exigió que el país firmara el TNP para continuar con la transferencia de material sensible, lo que generó ciertas dificultades para la transferencia de tecnología CANDU. ${ }^{32}$

30 Sotomayor, 2012, op. cit.

${ }^{31}$ Hurtado, op. cit.

32 Canadian Deuterium Uranium, es una marca registrada de la Empresa AECL que caracteriza a los reactores de agua pesada y uranio natural fabricados por ella. Véase Domingo Quilici: "Desarrollo de proveedores para la industria nuclear argentina. Visión desde las centrales nucleares”, Revista de historia de la industria argentina y latinoamericana, 2, núm. 2 (2008), pp. 2-24. 
Que Argentina se hubiera negado a ratificar el TNP y Tlatelolco en cierta forma la convirtió en un Estado umbral, ${ }^{33}$ situación que sin embargo la obligó a desarrollar sus propias soluciones tecnológicas, como fue evidente con la construcción secreta de una planta de enriquecimiento de uranio en Pilcaniyeu. La CNEA y la cancillería adoptaron una política conjunta para dar forma a una "diplomacia nuclear"34 en nichos no explotados en países miembro del Movimiento de los No Alineados (NOAL) como Yugoslavia, India, Argelia, de forma tal que se erigió como uno de los pocos PED exportadores de tecnología nuclear a otros países de nivel de desarrollo parecido que, además, no imponía exigencias de seguridad excesivas.

\section{El caso de México}

México pareciera ser un ejemplo contrario a prácticamente todas las decisiones tomadas en el caso anterior. Para algunos, su desarrollo científico y tecnológico siempre ha estado supeditado a dinámicas externas "que no se corresponden cabalmente con las condiciones de la actividad científica y tecnológica local ni con necesidades internas de la planta productiva". ${ }^{35}$ Aquí es clara una primera diferencia con el caso argentino: en México, no se habrían concebido los desarrollos nucleares como parte de un plan estratégico de la agenda científico-tecnológica, sino como consecuencia de las tendencias internacionales y de agendas foráneas de investigación. Desde esta perspectiva sería entonces comprensible que el programa atómico no se haya convertido en una

${ }^{33}$ Aquel con capacidad tecnológica para desarrollar armas nucleares, pero cuyas intenciones no son claras.

${ }^{34}$ Véase David Sheinin, "Nuclear development and the shaping of an Independent Argentine Foreign Policy (1950-1990)”, E.I.A.L, 16, núm. 2 (2005).

${ }^{35}$ Raúl Domínguez Martínez, "Los orígenes de la física nuclear en México”, Revista CTS, 7, núm. 21 (2013), pp. 95-112. 
política de Estado, en el sentido de ser una política pública que supere los sexenios presidenciales y que genere tradiciones científicas locales como las que se mostraron en el caso anterior.

Otras lecturas sugieren que sí hubo una consideración hacia las posibles contribuciones del plan nuclear al desarrollo del país, pues se sostenía que el grado de complejidad de esta tecnología implicaría el desarrollo de capacidades técnicas, científicas, profesionales e institucionales que servirían como plataforma a todo el sistema de investigación y desarrollo. ${ }^{36}$ Sin embargo coinciden en que el proyecto nuclear mexicano enfrentó las carencias y dificultades propias de un PED, donde tuvieron impacto las rupturas políticas sexenales debido tanto a la sucesiva recomposición de los grupos de poder como a sus percepciones de la cyт y los programas de política energética.

El programa nuclear mexicano nació a mediados de la década de 1950, con la cooperación de Estados Unidos, a través del Programa Átomos para la Paz. Las relaciones con el país del norte se veían desde un prisma positivo -siempre y cuando permitieran a México mantener su autonomía-, y su cooperación en términos científicos también se consideró como una oportunidad para alentar cierta industrialización por sustitución de importaciones. ${ }^{37}$ A partir de ese momento, con la participación de la compañía Mexicana de Luz y Fuerza Motriz, se enviaron a varios estudiantes a cursar ingeniería nuclear a Estados Unidos, quienes a su regreso integraron la CNEN, instaurada el $1^{\circ}$ de enero de $1956 .{ }^{38}$ Para algunos autores ${ }^{39}$ ése fue uno de los factores que propicia-

${ }^{36}$ Luz Azuela y José Luis Talancón, Contracorriente: historia de la energía nuclear en México (1945-1995), México, Plaza y Valdés, 1999.

37 Domínguez Martínez, art. cit.

38 Véase Dimitri Stevis y Stephen Mumme, "Nuclear Power, Technological Autonomy, and the State in Mexico", Latin American Research Review, 26, núm. 3 (1991), pp. 55-82.

39 Domínguez Martínez, art. cit. 
ron que la industria nuclear mexicana quedara supeditada a la estadounidense.

No obstante, los objetivos principales del programa nuclear de México tuvieron varios puntos de contacto con los del programa argentino, entre los cuales pueden mencionarse la diversificación energética, la transferencia de tecnología, la búsqueda de prestigio internacional y, por supuesto, la autonomía tecnológica. El primero tuvo especial importancia puesto que implicaba buscar un sustituto al petróleo, del que el país era muy dependiente. ${ }^{40}$ A pesar de lo anterior, muchas decisiones parecieron extraviar los caminos del entramado nuclear mexicano, como tratará de explicarse en adelante.

\section{Autonomía lateral}

La CNEN, al igual que la CNEA en Argentina, centralizó formalmente casi todas las tareas y funciones relacionadas con lo atómico. Pero mientras que en Argentina esta centralización permitió que la institución gozara de autonomía lateral, y mantuviera la concentración del proceso decisorio, en México fue visto como un lastre, resultando en "un cúmulo heterogéneo de propósitos, pautas e incluso de profesionistas, a los cuales tampoco se les fijaron metas y objetivos claramente delineados mediante un programa específico que plasmara el perfil de nuestras pretensiones nacionales en materia de desarrollo nuclear". ${ }^{41}$

Y aunque la CNEN mantuvo durante dos décadas tal concentración de tareas, su autonomía formal se vio desafiada por la Comisión Federal de Electricidad (CFE) que orbitó el programa nuclear en lo que respecta a los aspectos sobre la generación de nucleoelectricidad comercial, contando in-

${ }^{40}$ Stevis y Mumme, art. cit.

41 David Sarquís Ramírez, "Apuntes para la historia de la ciencia y la tecnología nuclear en México”, Revista Multidisciplina, 15 (mayo-agosto de 2013), pp. 141-142. 
cluso con una comisión de ingeniería nuclear para analizar la eventual utilización de electricidad nuclear en México. ${ }^{42}$ En el grupo de toma de decisiones formado para aquella tarea se encontraron representantes tanto de la empresa Petróleos Mexicanos (Pemex) como del Instituto de Investigaciones de Stanford (Estados Unidos), pero ninguno de la CNEN, lo que mostraba su rol marginal en la toma de decisiones.

La centralización formal de tareas duró hasta 1979, cuando la GNEN (devenida en Instituto Nacional de Energía Nuclear -INEN- en 1972) fue dividida en Uranios Mexicanos (Uramex, a cargo de todo lo relativo al ciclo del combustible nuclear y su comercialización), el Instituto Nacional de Investigaciones Nucleares (ININ, a cargo de la investigación y desarrollo de la tecnología nuclear) y la Comisión Nacional de Seguridad Nuclear y Salvaguardias (CNSNS, a cargo de los aspectos normativos y de supervisión en las aplicaciones de la energía nuclear con fines pacíficos). Esto derivó en una mayor pérdida de autonomía, ya que ahora no sólo debía competir contra la influencia de la CFE sino que además debía lograrse una coordinación entre tres agencias distintas para tomar decisiones en materia nuclear.

Por otra parte, en México también tuvo lugar una discusión sobre los caminos tecnológicos y sobre el tipo de tecnología a adoptar en relación con los reactores de potencia planificados. En un principio, al igual que la CNEA, los científicos de la CFE planificaron adquirir el "saber hacer" necesario para su propio programa, a través de la transferencia tecnológica. De esta forma, en lugar de apostar a la compra de un reactor llave en mano, priorizaron la opción de la compra de componentes que garantizaran transferencia de conocimientos a través del trabajo conjunto entre los ingenieros mexicanos y los contratistas extranjeros. Sin embargo, la CFE se inclinó hacia la tecnología de uranio enriquecido, alegando que era económicamente más ventajosa y más conocida. ${ }^{43}$

${ }^{42}$ Stevis y Mumme, art. cit.

${ }^{43}$ Loc. cit. Sarquís Ramírez, art. cit. 
La CFE se vio confrontada con los científicos del ININ, que eran favorables al uranio natural y al agua pesada puesto que " $[\ldots]$ se pensaba que no iba a comprometer la soberanía nacional al evitar la dependencia de la tecnología del uranio enriquecido dominada y promovida comercialmente en ese entonces principalmente por los Estados Unidos". 44 La victoria de la elección tecnológica de la CFE por sobre la de ININ dice mucho acerca del ambiente de segmentación lateral en que ambas agencias operaron, y también de las agendas prevalecientes.

En su rol central en la construcción de Laguna Verde -la primera central de potencia-, la CFE muchas veces enfrentó la crítica de los científicos nucleares, sobre todo debido a que, a diferencia del caso argentino, pocos sectores se vieron beneficiados por la transferencia de conocimiento ya que la tecnología adquirida fue casi monopolizada por la CFE y no transferida a otros sectores industriales ni al ININ. ${ }^{45}$

De esta forma, mientras Argentina comenzaba a exportar reactores y a desarrollar procesos para el enriquecimiento del uranio en forma secreta, en México la aspiración de alcanzar la autonomía tecnológica perdió gravitación por desacuerdos interagencias y las decisiones de la CFE de favorecer tecnologías y técnicos foráneos. ${ }^{46}$ Todo ello redundó en poca vinculación con el medio, sobre todo el productivo, escasos resultados para mostrar a la sociedad y la imposibilidad de explotar el rol autolegitimador de la incipiente industria nuclear mexicana.

Éste fue un caso típico de lo que se denominó segmentación lateral, ${ }^{47}$ basada en la constante competencia entre agencias con la consecuente imposibilidad de establecer un proyecto claro y unívoco. En el sector nuclear mexicano nunca se logró llegar a un acuerdo sobre objetivos básicos, como el

\footnotetext{
${ }^{44}$ Sarquís Ramírez, ibid., p. 163.

45 Stevis y Mumme, art. cit.

${ }^{46}$ Loc. cit.

47 Solingen, art. cit.
} 
tipo de tecnología a utilizar o el rol de la energía nuclear en el sistema energético mexicano, sino que existieron agendas "ganadoras" y "perdedoras", producto de la competencia entre organismos del área. Es evidente que en México las agencias dedicadas a lo nuclear debieron enfrentar el poco peso decisorio que determinó su reducida autonomía lateral, tanto por una división de tareas que impidió la coordinación de decisiones, como por el poco apoyo institucional que recibió de las presidencias mexicanas.

\section{Alianzas con actores intermediarios clave}

A diferencia de la CNEA, que contó con un fuerte respaldo y protección de la Marina, la CNEn no tuvo aliados domésticos de tanto peso. Fueron los propios científicos del área nuclear, haciendo uso de su capital social, prestigio internacional y de sus conexiones, quienes actuaron como los intermediarios entre sus proyectos atómicos y las altas esferas de decisión. Sin embargo, tal capacidad de intermediación demostró ser inestable y poco duradera y, aunque arrojó resultados concretos, no permitió modificar la orientación del programa hacia políticas percibidas como más conducentes a la autonomía tecnológica.

Efectivamente, los expertos nucleares mexicanos estaban muy bien relacionados con sus pares estadounidenses y de otras latitudes. Así lo demuestran varios hechos, surgidos de las biografías de expertos como Manuel Sandoval Vallarta y su influencia en la profesionalización e institucionalización de la física en México, en su puesto en el Massachusetts Institute of Technology (MIT). Desde la Segunda Guerra Mundial, en el contexto de la "política de la buena vecindad", había sido uno de los principales colaboradores con el gobierno estadounidense "en el establecimiento de mecanismos de intercambio entre científicos de América Latina” y 
de Estados Unidos. ${ }^{48}$ También tuvo influencia en la instalación de una estación para medir rayos cósmicos con asistencia del Nobel de Física estadounidense, Arthur Compton; y realizó investigaciones y publicaciones con Georges Lemaitre, lo que le valió el reconocimiento internacional. La gran consideración que se le tenía con base en su prestigio y conocimientos quedó patente además en su participación, en la década de 1940, en las negociaciones internacionales en la onU para el establecimiento de lo que más tarde sería la oieA, una de las razones por las que fue catalogado como "científico diplomático" ${ }^{49}$ Esto último le permitió convertirse en asesor de los gobiernos mexicanos y en representante del país en materia nuclear. Hacia 1951, en plena construcción de Ciudad Universitaria, el prestigio internacional de Sandoval Vallarta "era utilizado para validar la calidad de la investigación universitaria". ${ }^{50}$ Otros como Nabor Carrillo Flores se encontraban muy conectados con instituciones estadounidenses, tales como la Universidad de Harvard o la Fundación Rockefeller.

Dos ejemplos sirven para mostrar la importancia de estas alianzas en la concreción de metas específicas del programa nuclear mexicano. El primero es con respecto al proyecto de instalación de un reactor de investigación en la CNEN. Este proyecto se urdió en la década de 1960, en un contexto muy contradictorio para los avances nucleares. México, bajo la presidencia de López Mateos, se encontraba, por un lado, ante la encrucijada planteada por la crisis de los misiles, que alentó el TdT (ver sección siguiente) y, por el otro, ante la oportunidad percibida por el científico nuclear Carlos Graef al ser designado representante mexicano ante la Junta de

48 Adriana Minor y Joel Vargas Domínguez, "La incursión del científico diplomático en el siglo xx: dos experiencias mexicanas", Revista Digital Universitaria, 16, núm. 4 (2015), p. 10.

${ }^{49} \mathrm{Ibid}$.

${ }^{50}$ Gisela Mateos, Adriana Minor y Valeria Sánchez, "Una modernidad anunciada: la historia del Van de Graaf de Ciudad Universitaria", en Historia mexicana, 62, núm. 1 (2012), p. 434. 
Gobernadores de la oIEA, de gestionar un convenio de cooperación con el organismo para obtener un reactor de investigación ${ }^{51}$. Ante la negativa de López Mateos de tratar el tema, debido a que consideraba contradictorio el avance en ciertas áreas atómicas en el plano doméstico y su política exterior en materia nuclear, ${ }^{52}$ Carrillo Flores se encargó de convencer a los miembros de su gabinete para presentarle el proyecto, que más tarde contó con el apoyo del presidente y pudo materializarse.

El segundo ejemplo de la utilidad de las redes de cooperación tejidas por los expertos nucleares en el impulso del programa nuclear tiene que ver con la finalización de la central de Laguna Verde. Frente a constantes embates de grupos civiles opuestos a la instalación de la usina por consideraciones ambientales, de seguridad y por acusaciones de corrupción en el proyecto, el gobierno mexicano acudió en la década de 1980 a la OIEA, para que ésta realizara una inspección profunda de la planta y la declarara segura. De esta forma, ciertos autores atribuyen la conclusión de la obra y su posterior puesta en marcha ${ }^{53}$ no tanto a alianzas con actores domésticos, sino a la legitimación otorgada por el organismo internacional, con el cual los expertos atómicos mexicanos estaban muy bien conectados.

\section{Política exterior mexicana en materia nuclear}

En su política exterior, México se caracterizó por su vocación pacífica y su adhesión a los lineamientos internacionales. A diferencia de Argentina, fue el país que propuso la creación de la primera Zona Libre de Armas Nucleares (ZLAN) a través de la firma del TdT y se adhirió al TNP sin grandes reservas.

${ }^{51}$ Azuela y Talancón, op.cit.

${ }^{52}$ Loc. cit.

${ }^{53}$ Stevis y Mumme, art. cit. 
Sin embargo es necesario destacar que al momento de formar parte de la OIEA, en la segunda mitad de la década de 1950, el representante permanente de México ante la onU, Rafael de la Colina, expresó lo siguiente al secretario de Relaciones Exteriores:

No se alcanzaron los objetivos principales que perseguíamos, debido a la insistencia de las potencias más adelantadas en la tecnología de la energía atómica, de guardar para sí una situación privilegiada, análoga en algunos aspectos a la que tienen los Miembros Permanentes del Consejo de Seguridad. De hecho, es fácil observar hasta qué grado se repitieron en Washington las maniobras realizadas en San Francisco para restar fuerza y autoridad al Órgano plenamente representativo de la nueva entidad. Las consabidas frases [igualdad soberana de todos los miembros], [debido respeto a los derechos soberanos de los Estados], etc., no alcanzan a disfrazar el verdadero sentido del proyectado convenio, o sea, el de robustecer el dominio de las llamadas potencias atómicas. ${ }^{54}$

De la cita anterior se desprende que la diplomacia y la burocracia mexicanas eran conscientes de la situación de desigualdad derivada de las organizaciones y tratados del RINP, no obstante lo cual el país siempre apoyó las iniciativas de las potencias nucleares. Algunos autores consideran que México es un país con menor nivel de desarrollo nuclear en comparación con sus pares latinoamericanos, pero con alta disposición a mantener relaciones fluidas con Estados Unidos, a diferencia de Argentina, Brasil o Chile, que mostraron más limitaciones en su cooperación con Washington, ${ }^{55}$ ensayando dos posibles explicaciones de esta posición.

Primero, por sus tradiciones legalistas y basadas en principios en sus abordajes diplomáticos, una actitud emprendedora de México en materia de desarme que ha contribuido

${ }^{54}$ Citado en Domínguez Martínez, art. cit., p. 111.

${ }^{55}$ Véase Sotomayor, 2012, op.cit. 
a su prestigio internacional y estatura moral, en la búsqueda de generar confianza en Estados Unidos. En segundo lugar, es posible que su postura internacional principista haya sido complementada por el hecho de que su economía siempre fue más aperturista que aquella de Argentina, por ejemplo, y que por lo tanto sus estrategias de desarrollo hayan dependido enormemente del comercio y la cooperación internacional provenientes de la oiEa y de Estados Unidos. Por eso, la búsqueda de transparencia en sus políticas nucleares se erigió en una garantía para atraer inversionistas y flujos de cooperación. ${ }^{56} \mathrm{Si}$ bien lo anterior tuvo la ventaja de presentar al país como un receptor válido de cooperación científica y tecnológica exterior, y de interesar a posibles inversionistas en la estructura nuclear, también puede haber actuado en detrimento de los propios objetivos de independencia y autonomía energética. ${ }^{57}$

Por su parte, otros añaden que la dependencia mexicana frente a su meta de lograr desarrollar un programa nuclear propio tuvo en sus orígenes la política de no proliferación nuclear estadounidense y su política de seguridad nacional, factores de constreñimiento, tanto desde un punto de vista diplomático como científico-tecnológico. ${ }^{58}$ Esto quedó ejemplificado en la disputa en torno a los efectos de la Ley Carter de No Proliferación de 1978, que puso en peligro la importación de uranio enriquecido de Estados Unidos a México. Aunque esta situación fue resuelta con intermediación de la OIEA, atizó de alguna manera los debates en torno a la dicotomía dependencia-autodeterminación en el seno del sector nuclear, especialmente dentro del Sindicato Único de Trabajadores del INEN (sUTINEN), uno de los principales promotores de la defensa de la independencia y autonomía tecnológicas.

56 Loc. cit.

57 Ibid., pp. 26-27.

58 Rogelio Ruiz, "El sueño nuclear en el México de 1980", Nexos, 1 de octubre de 1987. 


\section{Comentarios finales}

Como se pudo observar a lo largo del artículo, los desarrollos nucleares en Argentina y México han seguido caminos muy distintos. Ya de por sí, el sector nuclear en Argentina tuvo una trayectoria particular, que prácticamente no es posible encontrar en ningún otro sector tecnológico del país. En su caso, la autonomía lateral de la CNEA otorgó mayor libertad para potenciar las ideas de autonomía tecnológica presente en la comunidad de expertos nucleares, lo cual obró en pos del aliento a la vinculación entre los sectores productivo y científico-tecnológico y en la creación de dinámicas convergentes, no sólo como prescripción de las ideas autonomistas, sino además como un mecanismo de autolegitimación frente a otros actores. Esta misma autonomía permitió también presentar a los responsables de tomar decisiones un abanico limitado de opciones tecnológicas, que se resumió en opciones de centrales de potencia alimentadas por uranio natural y agua pesada.

En México, por su parte, la centralización de tareas nucleares podría haber significado una gran autonomía lateral para la CNEN, pero ésta debió competir con la CFE, cuyas ideas resultaron, a pesar de sus esfuerzos en sentido inverso, en la búsqueda de eficiencia económica a corto plazo por sobre el esfuerzo de los desarrollos propios, incrementales y sostenidos. Al igual que en Argentina, estuvo presente el debate sobre el tipo de tecnología de reactores de potencia a adoptar, pero mientras los físicos y expertos de la CNEN/ININ mostraron mayor inclinación a la opción de uranio natural, la elección tecnológica terminó por decantarse en favor del uranio enriquecido, preferida por la CFE.

La existencia de estas discusiones demuestra que había consciencia sobre los fines de cada uno: mientras la tecnología de uranio al natural y agua pesada era más cara, disminuía la dependencia de proveedores externos y no estaba tan extendida, por lo que era posible que su comercialización encontrara menos trabas a nivel internacional, por el 
contrario, la tecnología más difundida de uranio enriquecido era más económica y eficiente, pero determinaba la dependencia de la provisión de uranio enriquecido de los únicos dos países que en ese momento podían suministrarlo: Estados Unidos y la Unión Soviética, que exigían el cumplimiento de varias salvaguardias y acuerdos internacionales que muchas veces se interpretaban como obstáculos a los desarrollos propios.

Con respecto a la segunda dimensión sobre alianzas con actores intermediarios claves, en Argentina es evidente la actuación de la Marina como catalizadora del proyecto atómico debido a dos cuestiones principales: primero, por la importancia que los militares mantuvieron como actores políticos durante las cuatro décadas posteriores a la Segunda Guerra Mundial, ya fuera ocupando directamente el Estado o tutelando a los gobiernos civiles. Esto permitió que los proyectos nucleares que ellos defendieron mantuvieran una presencia privilegiada en las agendas de políticas públicas, y su actuación como intermediarias de los intereses de la comunidad científica nuclear siempre que los desarrollos fueran pacíficos. Esta alianza estable y duradera durante más de tres décadas permitió la supervivencia del sector atómico, incluso durante la última dictadura cívico-militar de 19761983, que implementó las primeras medidas neoliberales en Argentina.

Segundo, la alianza con este actor $-y$ no con otro- demostró ser crucial por la importancia que el desarrollo nuclear revestía para éste en un contexto de posguerra y de especulación sobre una posible Tercera Guerra Mundial. En tal escenario, la autonomía e independencia tecnológica y energética se constituyó en un objetivo primordial para asegurar la supervivencia del Estado y su soberanía. En este marco, la tecnología atómica tenía un papel fundamental en la generación energética y como alentadora de una vinculación exitosa con la industria.

Por su parte, en México, debido a la existencia de una democracia institucionalmente estable desde mediados de la 
década de 1930 con un sistema de partido único, las Fuerzas Armadas no participaron de decisiones políticas en general, ni nucleares en particular, ya fuera en el ámbito doméstico o internacional, incluso a pesar de la amenaza que planteó la crisis de los misiles para el país. ${ }^{59}$ En su lugar, la comunidad de expertos nucleares acudió a diversos actores para concretar sus proyectos (miembros de gabinete, organismos internacionales) y a su propio capital social, pero estas alianzas parecieran haber sido ad hoc, y no hay indicios de que hayan resultado ser vehículos concretos para la potenciación e implementación de las ideas de autonomía tecnológica. Más bien sirvieron para dar respuestas a demandas específicas, como lo muestran los casos del reactor del Centro Salazar y la finalización de Laguna Verde.

En cuanto a la última dimensión, también hubo marcadas diferencias entre ambos países: mientras México hizo de la política de no proliferación su carta de presentación al sistema internacional, Argentina mantuvo una política de no adhesión al TdT ni al TNP por considerarlos injustos y discriminatorios. Aunque esta postura dificultó muchas transacciones a nivel internacional, obligó al país a pensar sus propias estrategias y le dio mayor ímpetu para avanzar con sus desarrollos. Por su parte, en México, la crisis de los misiles y la puesta en marcha de las negociaciones para el TdT significaron una gran contradicción para López Mateos con respecto al programa atómico doméstico. ${ }^{60}$ No es extraño, de esta forma, que en la primera mitad de la década de 1980, Argentina haya podido enriquecer uranio, logrando el control de la totalidad del ciclo del combustible nuclear, mientras que alrededor de esa misma época México cerraba Uramex.

Entonces, retornando a lo mencionado en el apartado teórico-metodológico sobre las condiciones que deben cumplir las explicaciones en torno a ideas, es posible concluir que en ambos casos puede trazarse la existencia de la idea de au-

${ }^{59}$ Sotomayor, 2014, art. cit.

60 Azuela y Talancón, op.cit. 
tonomía -o autodeterminación- tecnológica. Los responsables de tomar decisiones nucleares en CNEA y CNEN tenían convicciones originadas en la idea de autonomía tecnológica, que no se redujeron a cuestiones materiales (como lo ilustra el hecho de que en ambos casos hubiera predilección por los reactores de uranio natural). Sin embargo, las diferencias se plasman en los valores de las dimensiones que permitieron su vehiculización, potenciación y cristalización en políticas públicas concretas.

Por lo tanto, retomando la hipótesis planteada en la introducción del trabajo, puede afirmarse que el mayor desarrollo alcanzado por Argentina (registrado en el cuadro 1) en un lapso de poco más de cuatro décadas, se debió a la interacción de la autonomía lateral de la CNEA, sumada a la efectiva alianza estable y duradera con la Marina y a una política exterior orientada a la defensa del desarrollo autónomo, factores que permitieron ampliar la capacidad de la CNEA de implementar opciones tecnológicas conducentes a la autonomía tecnológica. En cambio en México, aunque la idea de autodeterminación tecnológica estuvo presente entre los miembros de la comunidad nuclear, la segmentación lateral, la existencia de alianzas ad hoc para metas específicas y una política exterior en materia nuclear más alineada al régimen de no proliferación resultaron, en el largo plazo, en un menor avance del sector.

\section{BibLIOGRAFÍA}

AdLER, Emanuel, "State Institutions, Ideology, and Autonomous Technological Development: Computers and Nuclear Energy in Argentina and Brazil”, Latin American Research Review, 23 (2), 1987, pp. 59-90.

Azuela, Luz y José Luis Talancón, Contracorriente: historia de la energía nuclear en México (1945-1995), México, Plaza y Valdés, 1999. 
Buzan, Barry y George Lawson, The Global Transformation: History, Modernity, and the Making of International Relations, Cambridge, University Press, 2015.

Carasales, Julio, La cooperación internacional de la Argentina en el campo nuclear, Buenos Aires, CARI, 1999.

Collier, David, "Understanding Process Tracing", Political Science and Politics, 44 (4), octubre de 2011, pp. 823-830.

Dagnino, Renato, Hernán Thomas y Amílcar Davyt, "El pensamiento en ciencia, tecnología y sociedad en Latinoamérica: una interpretación política de su trayectoria”, Revista REDES, 3 (7), septiembre de 1996, pp. 13-53.

Domínguez Martínez, Raúl, "Los orígenes de la física nuclear en México”, Revista cTS, 21 (7), 2013, pp. 95-112.

Dunn, Lewis, “The NPT”, The Nonproliferation Review, 16 (2), 2009, pp. 143-172.

GADANO, Julián, "El desarrollo nuclear pacífico argentino: factores que lo hicieron posible y algunas hipótesis sobre su futuro", En Joint International Conference 'Global and regional powers in a changing world', Buenos Aires, Flacso-IsA, 10 de julio de 2014, pp. 47-66.

Gerring, John, Case study research. Principles and practices, Cambridge, University Press, 2007.

GiLPIn, Robert, War and change in world politics, Cambridge, University Press, 1981.

Hurtado, Diego, El sueño de la Argentina atómica. Política, tecnología nuclear y desarrollo nacional (1945 - 2006), Buenos Aires, Editorial Edhasa, 2014.

Hymans, Jacques, "Of gauchos and gringos: Why Argentina never wanted the bomb, and why the United States thought it did", Security Studies, 10 (3), pp. 153-185.

Jacobs, Alan, "Process-tracing the effect of ideas", en Andrew Bennett y Jeffrey T. Checkel (eds.), Process Tracing in the Social Sciences: From Metaphor to Analytic Tool, Cambridge, University Press, 2014.

Jones, Rodney, “Atomic Diplomacy in Developing Countries”, en Journal of International Affairs. International Relations of Developing Countries (primavera-verano de 1980), 34 (1), pp. 89-117. 
Krige, John y Kai-Henrik BARTH, "Introduction: Science, Technology, and International Affairs", Osiris, 21 (1), 2006, pp. 1-21.

Martínez Vidal, Carlos y Manuel Marí, "La escuela latinoamericana de pensamiento en ciencia, tecnología y desarrollo. Notas de un proyecto de investigación”, Revista iberoamericana de ciencia, tecnología, sociedad e innovación, núm. 4, septiembre-diciembre de 2002.

Mallea, Rodrigo, Matías Spektor y Nicholas Wheeler, Origens $d a$ cooperação nuclear. Uma historia oral critica entre Argentina e Brasil, Río de Janeiro, fGv, Escola de Ciências Sociais, 249 pp.

Mateos, Gisela, Adriana Minor y Valeria SÁnchez, "Una modernidad anunciada: historia del Van de Graaf de Ciudad Universitaria”, Historia mexicana, 62, núm. 1 (2012), pp. 415-442.

Minor, Adriana y Joel Vargas Domínguez, "La incursión del científico diplomático en el siglo xx: dos experiencias mexicanas", Revista Digital Universitaria, 16 (4) 2015, pp. 1-16.

Oszlak, Oscar, "Políticas públicas y regímenes políticos. Reflexiones a partir de algunas experiencias latinoamericanas", Documento de Estudio de CEDES, 3 (2), 1984.

Quilici, Domingo, "Desarrollo de proveedores para la industria nuclear argentina. Visión desde las centrales nucleares", Revista de Historia de la industria argentina y latinoamericana, 2 (2), 2008, pp. 2-24.

Ruiz, Rogelio, "El sueño nuclear en el México de 1980", Nexos, 1 de octubre de 1987.

SARquís Ramírez, David, "Apuntes para la historia de la ciencia y la tecnología nuclear en México", Revista Multidisciplina, 15, mayo-agosto de 2013, pp. 129-175.

Schmidt, Vivien, "Discursive Institutionalism: The Explanatory Power of Ideas and Discourse", Annual Review of Political Science, 11, 2008, pp. 303-326.

Sheinin, David, "Nuclear development and the shaping of an Independent Argentine Foreign Policy (1950-1990)", E.I.A.L., 16 (2), 2005.

Solingen, Etel, "Macropolitical Consensus and Lateral Autonomy in Industrial Policy: The Nuclear Sector in Brazil and Argentina”, International Organization, 47 (2), 1993, pp. 263-298. 
Sotomayor, Arturo, U.S.-Latin American Nuclear Relations: From Commitment to Defiance, California, U.S Naval postgraduate School, 2012.

Sotomayor, Arturo, "Brazil and Mexico in the Nonproliferation Regime, common structures and divergent trajectories in Latin America", en J. R. Fields (ed.), State Behavior and the Nuclear Nonproliferation Regime, EEUU, University of Georgia Press, 2014. Stevis, Dimitri y Stephen Mumme, "Nuclear Power, Technological Autonomy, and the State in Mexico", Latin American Research Review, 26 (3), 1991, pp. 55-82. 
